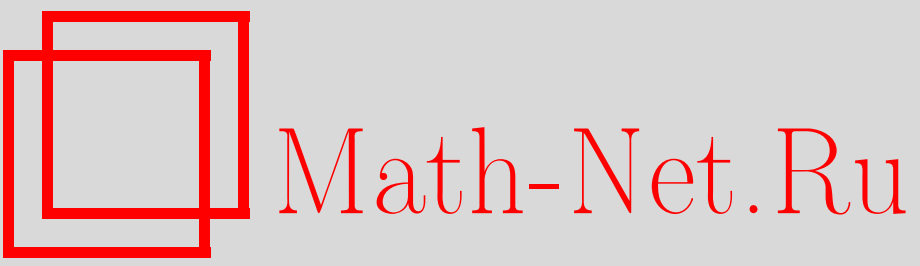

Л. А. Шепп, А. Н. Ширяев, Русский опцион в условиях возможного "замораживания" цен, УМН, 2001, том 56, выпуск 1, 187-188

DOI: https://doi.org/10.4213/rm377

Использование Общероссийского математического портала Math-Net.Ru подразумевает, что вы прочитали и согласны с пользовательским соглашением

http://www.mathnet.ru/rus/agreement

Параметры загрузки:

IP: 18.207 .199 .55

26 апреля 2023 г., 12:18:41 


\title{
РУССКИЙ ОПЦИОН В УСЛОВИЯХ ВОЗМОЖНОГО “ЗАМОРАЖИВАНИЯ" ЦЕН
}

\author{
Л. А. ШЕПП, А. Н. ШиРяЕВ
}

1. Модель производного финансового инструмента "Русский опцион", введенного авторами, $[1],[2]$, и относящегося к классу опционов (-пут, put option) с последействием и дисконтированием, описьвается следующим образом.

Пусть цены $X=\left(X_{t}\right)_{t \geqslant 0}$ некоторого финансового актива задаются геометрическим броуновским движением

$$
X_{t}=x \exp \left(\sigma B_{t}+\left(r-\frac{\sigma^{2}}{2}\right) t\right), \quad t \geqslant 0,
$$

где $B=\left(B_{t}\right)_{t \geqslant 0}$ - стандартное броуновское движение, $x>0, \sigma>0$, процентная ставка $r \geqslant 0$.

Обозначим $S_{t}=s \vee \max _{u \leqslant t} X_{u}, 0<x \leqslant s$, и положим для $\lambda>0$

$$
V^{*}(x, s)=\sup _{\tau \in \mathfrak{M}} \mathrm{E}_{x, s} e^{-(\lambda+r) \tau} S_{\tau}
$$

где $\mathfrak{M}$ есть класс конечных (Р $x, s$-п.н.) моментов остановки $\tau$ (относительно потока $\sigma$-алгебр, порожденных броуновским движением), $\mathrm{E}_{x, s}$ - усреднение по мере $\mathrm{P}_{x, s}$ - распределению вероятностей процесса $(X, S)=\left(X_{t}, S_{t}\right)_{t \geqslant 0}$ при $X_{0}=x, S_{0}=s$.

Нахождение функции (цены) $V^{*}(x, s)$ и соответствующего оптимального момента остановки $\tau^{*}$, на котором в $(2)$ достигается значение $V^{*}(x, s)$, представляет интерес в связи с расчетами рациональной (справедливой) цены, которую должен заплатить покупатель за приобретение опциона (Американского типа) с платежной функцией $f_{\tau}=\max _{u \leqslant \tau} S_{u}$, когда опцион предъявляется (покупателем) к исполнению в момент времени $\tau$. (См. подробнее [3; гл. I, $\S 1 \mathrm{c} ;$ гл. VIII, $\S 2 \mathrm{~d}]$.

В [1], [2] был получен следующий результат.

Пусть $A=1-2 r / \sigma^{2}, B=2(\lambda+r) / \sigma^{2}$, и пусть $\gamma_{k}=A / 2+(-1)^{k} \sqrt{(A / 2)^{2}+B}, k=1,2,-$ два решения $\left(\gamma_{1}<0, \gamma_{2}>1\right)$ квадратного уравнения $\gamma^{2}-A \gamma-B=0$. Обозначим

$$
\theta=\left(\frac{1-\gamma_{1}^{-1}}{1-\gamma_{2}^{-1}}\right)^{1 /\left(\gamma_{2}-\gamma_{1}\right)} \quad(>1)
$$

и $g^{*}(s)=s / \theta, 0<x \leqslant s$. Тогда цена $V^{*}(x, s)$ и оптимальный момент остановки $\tau^{*}$ определяются формулами

$$
V^{*}(x, s)= \begin{cases}\frac{s}{\gamma_{2}-\gamma_{1}}\left[\gamma_{2}\left(\frac{x}{g^{*}(s)}\right)^{\gamma_{1}}-\gamma_{1}\left(\frac{x}{g^{*}(s)}\right)^{\gamma_{2}}\right], & g^{*}(s)<x \leqslant s \\ s, & x \leqslant g^{*}(s)\end{cases}
$$

$$
\tau^{*}=\inf \left\{t: X_{t} \leqslant g^{*}\left(S_{t}\right)\right\}
$$

(См. рис. 1 , где $C^{*}=\{(x, s): s / \theta<x \leqslant s\}$ - область продолжения наблюдений и $D^{*}=$ $\{(x, s): x \leqslant s / \theta\}$ - область остановки наблюдений.)

2. Цель настоящей заметки - рассмотреть некоторую интересную с нашей точки зрения модификацию "Русского опциона". Читатель сразу увидит, что предлагаемая модификация имеет простую финансово-экономическую интерпретацию, связанную с возможньм "замораживанием" цен, дефолтом, банкротством и т. п. С точки зрения общей теории оптимальных правил остановки речь будет идти о задаче с ограничением на допустимый класс моментов остановки.

Пусть $T_{\varepsilon}=\inf \left\{t \geqslant 0: X_{t} \leqslant \varepsilon\right\}, \mathfrak{M}_{\varepsilon}=\left\{\tau \in \mathfrak{M}: \tau \leqslant T_{\varepsilon}\right\}-$ класс моментов остановки из $\mathfrak{M}$, меньших или равных $T_{\varepsilon}$. Положим для $\lambda>0$

$$
V_{\varepsilon}^{*}(x, s)=\sup _{\tau \in \mathfrak{M}_{\varepsilon}} \mathrm{E}_{x, s} e^{-(r+\lambda) \tau} S_{\tau} .
$$

Ясно, что $V_{\varepsilon}^{*}(x, s)=\sup _{\tau \in \mathfrak{M}} \mathrm{E}_{x, s} e^{-(r+\lambda) \tau} S_{\tau} I\left(\tau \leqslant T_{\varepsilon}\right)$. Если ввести “остановленный" процесс $X_{t}^{\varepsilon}=X_{t \wedge T_{\varepsilon}}$, который можно интерпретировать как процесс, описьвающий "замороженные" цены, то видим, что $V_{\varepsilon}^{*}(x, s)=\sup _{\tau \in \mathfrak{M}} \mathrm{E}_{x, s} e^{-(r+\lambda) \tau} S_{\tau}^{\varepsilon}$, где $S_{\tau}^{\varepsilon}=s \vee \max _{u \leqslant t} X_{u}^{\varepsilon}$. 


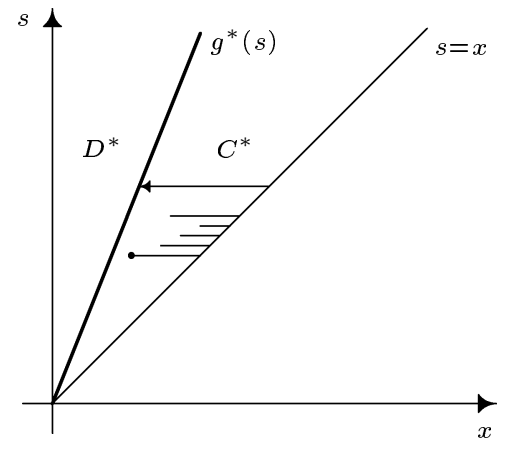

Рис. 1. "Русский опцион": $D^{*}-$ область остановки, $C^{*}$ - область продолжения наблюдений, $g^{*}(s)=s / \theta$ - граница области остановки (как функция от $s$ ) - выделена полужирным шрифотом

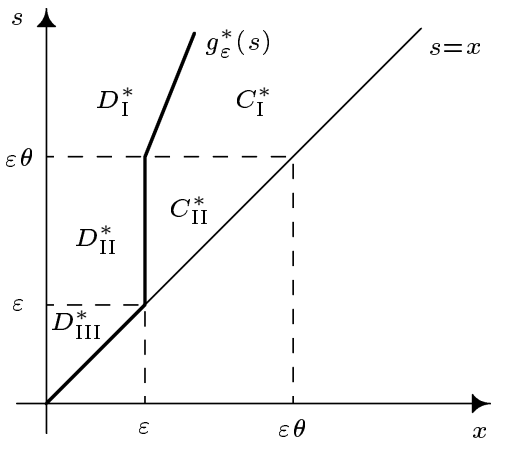

Рис. 2. Модифицированный "Русский опцион": $D^{*}-$ область остановки, $C_{\mathrm{I}}^{*} \cup C_{\mathrm{II}}^{*}-$ область продолжения наблюдений,

$g_{\varepsilon}^{*}(s)= \begin{cases}\max (\varepsilon, s / \theta), & \varepsilon \leqslant x \leqslant s, \\ s, & x<\varepsilon,\end{cases}$

- граница области остановки (как функция от $s$ ) - выделена полужирным шрифотом

Заметим, что для всякого $\varepsilon>0 V_{\varepsilon}^{*}(x, s) \leqslant V(x, s)$. Поскольку для всех $x>\varepsilon$ и $s>\varepsilon \theta$ для момента $\tau^{*}$, определенного в (5), выполнено свойство $\tau^{*}<T_{\varepsilon}\left(\mathrm{P}_{x, s}\right.$-П.н.), то для таких $(x, s) V_{\varepsilon}^{*}(x, s)=V(x, s)$ и момент $\tau^{*}$, оптимальный в задаче $(2)$, является также оптимальным в модифицированной задаче $(6)$.

Понятно также, что для всех $0<x<\varepsilon$ оптимальньй момент $\tau_{\varepsilon}^{*}=0$ и в этом неинтересном случае $V_{\varepsilon}^{*}(x, s)=s$ для всех $s \geqslant x$.

3. Положим $g_{\varepsilon}^{*}(s)=\max (\varepsilon, s / \theta)$.

ТЕорема. В модифицированной версии "Русского опциона" оптимальный момент остановки $\tau_{\varepsilon}^{*}=\inf \left\{t \geqslant 0: X_{t} \leqslant g_{\varepsilon}^{*}\left(S_{t}\right)\right\}$, если $\varepsilon \leqslant x \leqslant s, u \tau_{\varepsilon}^{*}=0$, если $x \leqslant s \leqslant \varepsilon$.

В области остановки $D^{*}=D_{\mathrm{I}}^{*} \cup D_{\mathrm{II}}^{*} \cup D_{\mathrm{III}}^{*}$, где $D_{\mathrm{I}}^{*}=\left\{(x, s): x \leqslant g^{*}(s), s \geqslant \varepsilon \theta\right\}$, $D_{\mathrm{II}}^{*}=\{(x, s): x \leqslant \varepsilon, \varepsilon \leqslant s \leqslant \varepsilon \theta\}, D_{\mathrm{III}}^{*}=\{(x, s): x \leqslant s \leqslant \varepsilon\}$ (cм. puс. 2), чена $V_{\varepsilon}^{*}(x, s)=s$.

В области продолжения наблюдений $C_{\mathrm{I}}^{*}=\left\{(x, s): g_{\varepsilon}^{*}(s)<x<s, s \geqslant \varepsilon \theta\right\}$ чена $V_{\varepsilon}^{*}(x, s)=V^{*}(x, s)$, где $V^{*}(x, s)$ определяется формулой (4). В области продолэения наблюдений $C_{\mathrm{II}}^{*}=\{(x, s): \varepsilon<x \leqslant s<\varepsilon \theta\} \quad V_{\varepsilon}^{*}(x, s)=A_{1}(s)(x / \varepsilon)^{\gamma_{1}}+A_{2}(s)(x / \varepsilon)^{\gamma_{2}}$, где

$$
A_{1}(s)=-\frac{\varepsilon}{\beta}\left[\int_{\beta \log (s / \varepsilon)}^{\beta \log \theta} \frac{e^{\left(\beta^{-1}+1\right) t}}{e^{t}-1} d t-\theta \gamma_{2}\right], \quad A_{2}(s)=\frac{\varepsilon}{\beta}\left[\int_{\beta \log (s / \varepsilon)}^{\beta \log \theta} \frac{e^{\beta^{-1} t}}{e^{t}-1} d t-\theta \gamma_{1}\right]
$$

$c \beta=\gamma_{2}-\gamma_{1}$.

Доказательство оптимальности момента $\tau_{\varepsilon}^{*}$ проводится с использованием "проверочных теорем", применяемых к функции $V_{\varepsilon}^{*}(x, s)$, находимой в свою очередь из решения соответствующей задачи Стефана.

\section{СПИСОК ЛИТЕРАТУРЫ}

[1] L. A. Shepp, A. N. Shiryaev // Ann. Appl. Probab. 1993. V. 3. №3. P. 631-640. [2] Л. А. Шепп, А. Н. Ширяев // Теория вероятн. и ее прим. 1994. Т. 39. №1. С. 130-149. [3] А. Н. Ширяев. Основы стохастической финансовой математики. М.: Фазис, 1998.

Rutgers University;

Математический институт им. В. А. Стеклова РАН,

Принято редколлегией

Московский государственный университет им. М. В. Ломоносова

01.12 .2000 